\title{
Nuclear spins as quantum memory in semiconductor nanostructures
}

\author{
W. M. Witzel and S. Das Sarma \\ Condensed Matter Theory Center, Department of Physics, \\ University of Maryland, College Park, Maryland 20742-4111, USA
}

(Dated: October 30, 2018)

\begin{abstract}
We theoretically consider the possibility of using solid state nuclear spins in a semiconductor nanostructure environment as long-lived, high-fidelity quantum memory. In particular, we calculate, in the limit of a strong applied magnetic field, the fidelity of $\mathrm{P}$ donor nuclear spins as a function of time in random bath environments of $\mathrm{Si}$ and GaAs, and the lifetime of excited intrinsic spins in polarized $\mathrm{Si}$ and $\mathrm{GaAs}$ environments. In the former situation, the nuclear spin dephases due to spectral diffusion induced by the dipolar interaction among nuclei in the bath; in the interest of the high fidelity requirements necessary for fault-tolerant quantum computing, we consider the initial quantum memory decay caused by a non-Markovian bath. We calculate this nuclear spin memory time in the context of Hahn and Carr-Purcell-Meiboom-Gill refocused spin echoes using a formally exact cluster expansion technique which has previously been successful in dealing with electron spin dephasing in a solid state nuclear spin bath. With decoherence dominated by transverse dephasing $\left(T_{2}\right)$, we find it feasible to maintain high fidelity (losses of less than $10^{-6}$ ) quantum memory on nuclear spins for times of the order of $100 \mu \mathrm{s}$ (GaAs:P) and 1-2 ms (natural Si:P) using CPMG pulse sequences of just a few $(\sim 2-4)$ applied pulses. We also consider the complementary situation of a central flipped intrinsic nuclear spin in a bath of completely polarized nuclear spins where decoherence is caused by the direct flip-flop of the central spin with spins in the bath. Exact numerical calculations that include a sufficiently large neighborhood of surrounding nuclei show lifetimes on the order of 1-5 ms for both GaAs and natural Si. Our calculated nuclear spin coherence times may have significance for solid state quantum computer architectures using localized electron spins in semiconductors where nuclear spins have been proposed for quantum memory storage.

PACS numbers: 03.67.-a; 76.60.Lz; 03.65.Yz; 76.30.-v; 03.67.Lx
\end{abstract}

\section{INTRODUCTION}

The motivation for developing a solid state quantum computer architecture using localized spins as qubits arises primarily from the long presumed quantum coherence times for spins even in the strongly interacting solid state environment. In this respect, nuclear spins are ideal since both spin relaxation (i.e., $\left.T_{1}\right)$ and spin coherence (i.e., $T_{2}$ ) times are very long for nuclear spins, as compared with electron spins, due to their weak coupling to the environment. The application of a strong magnetic field further enhances nuclear coherence by suppressing, at least, the leading order relaxation and decoherence processes caused by direct hyperfine coupling between nuclear spins and any surrounding electron spins due to the large mismatch between electron and nuclear spin Zeeman energies. Throughout this paper we assume a strong applied magnetic field that defines the $z$-direction of our interacting spin system, and we also neglect, somewhat uncritically, all effects of any direct hyperfine coupling between electron spins and nuclear spins assuming our system to be entirely a nuclear spin system. The existence of localized electron spins in the environment will further suppress the nuclear spin coherence, and therefore our theoretical values for nuclear spin quantum memory lifetimes should be taken as upper bounds.

Solid state nuclear spins as possible qubits and/or quantum memory have earlier been discussed in the literature $1,2,3,4,5$ in various contexts although we are not aware of any concrete calculations of spectral diffusion- induced nuclear spin decoherence using the cluster expansion technique (or any other quantitative techniques for that matter) along the line we carry out in this work. In particular, the vast NMR literature ${ }^{\underline{6}}$ (and the more recent literature on the prospective use of nuclear spins in the quantum information processing ${ }^{7}$ ) typically (and uncritically) assume exponential Markovian decay of nuclear spin quantum memory whereas we theoretically treat the non-Markovian nonexponential decay which dominates the initial decoherence of interest to quantum memory applications that require high fidelity. We emphasize in this context that we use the term $T_{2}$ to signify the time scale of the initial decay behavior (defined as the time in which the extrapolation of this initial decay behavior reaches $1 / e$ ). This is in sharp contrast to the ordinary definition of $T_{2}$ where an exponential decay is implicitly assumed.

The theoretical work presented in this paper deals entirely with the quantum memory of nuclear spins in solid state spin quantum computer architectures. In particular, we directly calculate solid state nuclear spin decoherence in the nuclear spin bath environment, i.e. for a Hamiltonian which contains only interaction between nuclear spins. Our work is of direct relevance to semiconductor-based quantum computer architectures where the idea of using nuclear spins to coherently store quantum spin memory has been seriously proposed in the literature $1.2,3,4.5$ As such, although we develop a rather general theoretical formalism for nuclear spin decoherence, we concentrate on GaAs quantum dots and phos- 
phorus doped silicon, Si:P, for applying our theory since much of the solid state quantum computer work in the literature has focused on these two architectures. 8

The theoretical question we address is simple and welldefined: How long is the (high-fidelity) solid state quantum memory time for nuclear spins in semiconductor structures? We answer this question in some detail for our specific model and systems, and discuss, using our formalism, how one can enhance the nuclear spin quantum memory (beyond the simple Hahn spin echo situation) by using composite pulse sequences to refocus nuclear spins. Our goal is thus a qualitative and quantitative understanding of nuclear spin quantum memory in GaAs quantum dots and Si:P doped structures taking into account the complex interacting environment of all the other nuclear spins invariably present in the solid state environment.

In considering nuclear spins as solid state quantum memory, we discuss two inequivalent (and perhaps complementary) situations. The usual situation is a bath of unpolarized nuclear spins surrounding the "central" donor nuclear spin which serves as our quantum memory. In this situation, spin relaxation processes $\left(T_{1}\right)$ are suppressed by applying a strong magnetic field assuming there is a significant mismatch between magnetic moments of the donor and intrinsic nuclei. Instead, the central spin memory dephases $\left(T_{2}\right)$ primarily through the spectral diffusion mechanism that is induced by the surrounding nuclear spin bath. In the spectral diffusion process for the high-fidelity (initial decay) regime that is our focus, the central spin quantum memory is effectively subjected to a non-Markovian temporally fluctuating magnetic field due to the nuclear spin flip-flops in the surrounding bath that result from dipolar interactions among the bath nuclei. The spectral diffusion, examined in Sec. III sets an ultimate limit to the coherence time for nuclear quantum memory storage in an unpolarized bath. There are, however, well-established procedures, going back fifty years,, 910 which can enhance (or more accurately restore) the quantum coherence over longer times. These coherence-enhancement protocols involve intricate refocusing of the nuclear spin dynamics through welldesigned composite pulse sequences. We discuss a particular kind of coherence enhancement protocol, namely the Carr-Purcell-Meiboom-Gill (CPMG) composite pulse sequence,,$\frac{9}{}$ in the context of spectral diffusion induced nuclear spin memory decoherence in Sec. III of this paper. In Sec. IV, we consider the complementary situation with quantum memory stored in a "central" intrinsic spin in a bath that is completely spin-polarized (perhaps through a dynamic nuclear polarization technique). This somewhat artificial situation (because complete nuclear spin polarization of the surrounding bath is highly improbable) is instructive because spectral diffusion is, by design, completely suppressed in a fully spin polarized nuclear spin bath (having no spin degrees of freedom available to cause fluctuations); relaxation, however, is now possible via direct dipolar flip-flops between the central nucleus and like nuclei (those with the same magnetic moment as the central nucleus and are thus still allowed to flip-flop in the limit of a strong applied magnetic field) in the bath. We solve this problem with exact quantum simulation (greatly simplified because of the complete polarization of the bath) and discuss the results in Sec. IV. We conclude in Sec. $\mathrm{V}$ with a discussion of the implications of our results for solid state quantum information processing and of the various limitations of our theory.

\section{SPECTRAL DIFFUSION OF A DONOR NUCLEUS}

Nuclear storage of quantum information in a solid state environment is most naturally placed on donor nuclei that are easily distinguishable from the surrounding intrinsic nuclei. It is imperative that the memory is stored in a nucleus which is distinct from the surrounding nuclei in some manner so that the stored information can be recovered. Several quantum computing architecture proposals $1,2,3,4$ exploit the long-term quantum information storage capabilities which donor nuclei spins can possess. In this section, we present theoretical calculations of the $T_{2}$ dephasing of donor nuclear spins in two solid state environments of interest for quantum computing: Si:P and GaAs:P. Specifically, we present coherence versus time information in the context of simple Hahn echo refocusing; in the next section, we address multiple pulse Carr-Purcell-Meiboom-Gill (CPMG) refocusing.

Fault-tolerant quantum computation demands high fidelity; quantum error correction typically requires $10^{4}$ to $10^{6}$ coherent operations 11 and therefore quantum memory loss should be kept below $10^{-4}$ to $10^{-6}$. The decoherence of nuclear spins has been well-studied in nuclear magnetic resonance (NMR) going back 50 years 6,12 and more recently $, 2,13$ However, these studies were not motivated by the need for such high fidelity. In NMR, it is typical and appropriate to assume Markovian-type decoherence, with an exponential decay form. However, we find that the initial $10^{-6}-10^{-4}$ part of the decay of nuclear spin coherence is non-Markovian (nonexponential). For our purposes, then, $T_{2}$ must take on a slightly different meaning than that of NMR. We find that the initial spectral diffusion decay will be of the form $\exp \left[-\left(t / T_{2}\right)^{n}\right]$, defining $T_{2}$ as a characteristic time scale for the initial decay (it is defined as the extrapolated $1 / e$ time of the decay although the decay will not generally match this extrapolation because it will eventually cross over into the Markovian regime with an exponential form). For considerations of high-fidelity quantum memory storage, the $n$ exponent in the exponential holds as much relevance as the $T_{2}$ time itself.

We have previously reported ${ }^{14}$ on a cluster expansion technique that is often applicable to spectral diffusion problems, giving formally exact solutions for short times. In some systems, formally exact solutions are obtained out to times that are longer than the decay time of 
the system; in such cases, the cluster expansion method solves the entire problem for practical purposes because the tail of the decay is of no consequence (assuming a monotonic decrease in coherence with time). Other systems, including the donor nucleus spin decay currently being investigated, yield convergent cluster expansion results for only the initial part of the decay. For this reason, and motivated by the strict high-fidelity requirements of quantum error correction, we focus on the initial decay and our plots show memory loss as a function of time rather than exhibiting full, formally exact, decay curves.

In our model, we take the limit of a strong applied magnetic field which completely suppresses any interactions that do not conserve Zeeman energy. For simplicity, we also disregard interactions with any electrons; our Hamiltonian is solely composed of interactions between nuclei. We consider only the spectral diffusion decoherence mechanism due to the dipolar flip-flops in the nuclear bath surrounding the central nuclear spin. The bath Hamiltonian consists of dipolar flip-flop interactions between like nuclei (as well as the diagonal $I_{n z} I_{m z}$ interaction):

$$
\begin{aligned}
\mathcal{H}_{B} & \approx \sum_{n \neq m} b_{n m}\left[\left\{\begin{array}{c}
I_{n+} I_{m-} \\
0
\end{array} \begin{array}{l}
\text { if } \gamma_{n}=\gamma_{m} \\
\text { otherwise }
\end{array}-2 I_{n z} I_{m z}\right],(1)\right. \\
b_{n m} & =-\frac{1}{4} \gamma_{n} \gamma_{m} \hbar \frac{1-3 \cos ^{2} \theta_{n m}}{R_{n m}^{3}}
\end{aligned}
$$

Here, $I_{n \pm}$ denote the raising and lowering operator for the $n$th nuclear spin and $\gamma_{n}$ its gyromagnetic ratio, $R_{n m}$ denotes the distance between nucleus $n$ and $m$, and $\theta_{n m}$ the angle of the vector between them relative to the applied magnetic field. Flip-flop interactions between unlike spins are suppressed via energy conservation due to the applied magnetic field.

The qubit-bath interactions are also due to dipolar coupling. (We refer to the central spin as a qubit to distinguish it from the nuclear spin bath.) However, in this instance we disregard the flip-flop interactions which are suppressed via energy conservation as a result of the applied magnetic field and differing gyromagnetic ratios between the qubit and spins in the bath. Instead, we consider just the $I_{n z} I_{m z}$ term which will contribute to dephasing:

$$
\begin{aligned}
\mathcal{H}_{A} & \approx \sum_{n} A_{n} I_{n z} S_{z}, \\
A_{n} & =\gamma_{\mathrm{D}} \gamma_{n} \hbar \frac{1-3 \cos ^{2} \theta_{n}}{R_{n}^{3}},
\end{aligned}
$$

where $S_{z}$ is a nuclear spin operator for the $\mathrm{P}$ donor nucleus, $\gamma_{\mathrm{D}}$ is the gyromagnetic ratio of the donor nucleus, $R_{n}$ is the distance of nucleus $n$ for the $\mathrm{P}$ donor, and $\theta_{n}$ is the angle of the vector from the $\mathrm{P}$ donor to nucleus $n$ relative to the applied magnetic field. Note that the $I_{n z} I_{m z}$ term of Eq. (11) requires an extra factor of $1 / 2$ to account for interchanging $n$ and $m$ that is not required in Eq. (3).
The free evolution Hamiltonian is given by $\mathcal{H}_{A}+\mathcal{H}_{B}$. We represent the Hahn echo sequence by $\tau \rightarrow \pi \rightarrow \tau$ which we take to mean: free evolution for a time $\tau$ followed by an NMR $\pi$-pulse rotation of the $\mathrm{P}$ donor nuclear spin about an axis perpendicular to the magnetic field, and then free evolution for another $\tau$ in time. The total time for this pulse sequence is $t=2 \tau$. In order to measure the decoherence of the $\mathrm{P}$ donor nuclear spin, we compute the magnitude of the expectation value of a $\mathrm{P}$ donor spin that is initialized perpendicular to the magnetic field (for maximal spectral diffusion) and evolves according to the Hahn echo sequence. When normalized to a maximum value of one, this expectation value, as a function of $\tau$, defines the Hahn echo envelope. Where $\langle\ldots\rangle$ is the quantum mechanical average over initial bath states, this Hahn echo envelope may be expressed as $\underline{14}$

$$
v_{\text {Hahn }}(\tau)=\left\langle\left[U_{\text {Hahn }}^{-}(\tau)\right]^{\dagger} U_{\text {Hahn }}^{+}(\tau)\right\rangle,
$$

where $U_{\mathrm{Hahn}}^{ \pm}(\tau)$ are nuclear evolution operators in the context of a Hahn echo sequence with an initially up $(+)$ or down (-) electron spin such that

$$
\begin{aligned}
U_{\text {Hahn }}^{ \pm}(\tau) & =U_{0}^{\mp}(\tau) U_{0}^{ \pm}(\tau) \\
U_{0}^{ \pm}(\tau) & =\mathrm{e}^{-\imath \mathcal{H}_{ \pm} t} \\
\mathcal{H}_{ \pm} & =\mathcal{H}_{B} \pm \frac{1}{2} \sum_{n} A_{n} I_{n z}
\end{aligned}
$$

assuming ideal $\pi$-pulses. Our calculations assume complete disorder of the initial nuclear spin bath states, expected in the large nuclear temperature limit $(T \gg m K)$ without dynamic nuclear polarization or some such technique to generate order; the quantum mechanical average, $\langle\ldots\rangle$, of Eq. (5) then simply averages over all possible nuclear states with equal weighting (that is, the trace of the nuclear spin operators divided by the number of nuclear spins states).

Using the formalism that we have laid out in Ref. 14, we can expand Eq. (5) in a way that includes contributions from "clusters" of successively increasing size. Defining $v_{\mathcal{C}}(\tau)$ as the solution to $v_{\text {Hahn }}(\tau)$ [Eq. (5)] when only considering those nuclei in some cluster (set) $\mathcal{C}$, a "cluster contribution" is recursively defined and computed by $\underline{14}$

$$
v_{\mathcal{C}}^{\prime}(\tau)=v_{\mathcal{C}}(\tau)-\sum_{\substack{\left\{\mathcal{C}_{i}\right\} \\ \mathcal{C}_{i} \neq \emptyset, \mathcal{C}_{i} \subset \mathcal{C}}} \prod_{i} v_{\mathcal{C}_{i}}^{\prime}(\tau),
$$

subtracting from $v_{\mathcal{C}}(\tau)$ the sum of all products of contributions from disjoint sets of clusters contained in $\mathcal{C}$. Defined in this way, a cluster contribution can only be significant when interactions between nuclei in the set are significant and no part is isolated from the rest. We consider only local dipolar interactions in the current work, and thus contributions only arise when the nuclei in the set are spatially clustered together; hence we refer to these sets as clusters. The $k$ th order of the cluster 
expansion for the echo [Eq. (5)] is then ${ }^{14}$

$$
v^{(k)}(\tau)=\sum_{\substack{\left\{\mathcal{C}_{i}\right\} \\ \mathcal{C}_{i} \neq \emptyset,\left|\mathcal{C}_{i}\right| \leq k}} \prod_{i} v_{\mathcal{C}_{i}}^{\prime}(\tau),
$$

and involves clusters of size no larger than $k$. To simplify the calculation, the logarithm of the echo may be approximated as

$$
\ln \left[v^{(k)}(\tau)\right] \approx \sum_{|\mathcal{C}| \leq k} v_{\mathcal{C}}^{\prime}(\tau)
$$

and corrections to this approximation, discussed in Ref. 14, may be computed to test its accuracy and are expected to be $O(1 / N)$, where $N$ is the number contributing spins in the bath.

We have performed such cluster expansion calculations to successively approximate Eq. (5) for two different systems. In both systems, we have a $\mathrm{P}$ donor atom with $\gamma_{\mathrm{D}}=\gamma_{\mathrm{P}}=1.08 \times 10^{4}(\mathrm{~s} \mathrm{G})^{-1}$, and we have chosen the applied magnetic field to point along one of the conventional axes directions (e.g., $B \|[001])$. In our figures, we plot "memory loss" versus total echo time $(2 \tau)$ where we define memory loss as one minus the echo envelope, $1-v_{\text {Hahn }}(\tau)$, and we only show results where the cluster expansion is rapidly convergent and the estimated correction to Eq. (11) is negligible. We show Hahn echo results (as well as CPMG results which will be discussed in the next section) for GaAs:P in Fig. 1] in this system, $\gamma_{n}=4.58,8.16$, and $6.42 \times 10^{3}(\mathrm{~s} \mathrm{G})^{-1}$ for ${ }^{75} \mathrm{As},{ }^{71} \mathrm{Ga}$, and ${ }^{69} \mathrm{Ga}$, respectively, and all differ from $\gamma_{\mathrm{P}}$. We additionally show Hahn echo results (and CPMG results) for Si:P in Figs. 2 and 3. $\gamma_{n}=5.31 \times 10^{3}(\mathrm{~s} \mathrm{G})^{-1}$ for ${ }^{29} \mathrm{Si}$ which also differs from $\gamma_{\mathrm{P}}$. Unlike GaAs, Si has stable isotopes $\left({ }^{28} \mathrm{Si},{ }^{30} \mathrm{Si}\right)$ with zero spin. Among its stable isotopes, only ${ }^{29} \mathrm{Si}$, which has a natural abundance of $4.67 \%$ and a spin of $1 / 2$, has a nonzero spin. Isotopic purification can reduce the amount of ${ }^{29} \mathrm{Si}$ and thereby diminish spectral diffusion caused by the nuclear spin bath. For generality, we define $f$ to be the fraction of Si that is the ${ }^{29} \mathrm{Si}$ isotope. Figure 2 shows results in a natural $\mathrm{Si}$ bath $(f=0.0467)$, while Fig. 3 shows, for comparison, results in a bath of Si isotopically purified to $f=0.01$.

As an alternative to the cluster expansion, a lowest order expansion of $v_{\mathrm{Hahn}}(\tau)$ [Eq. (5)] in $\tau$ reveals that $v_{\text {Hahn }}(\tau)=1-O\left(\tau^{4}\right)$, and this simple lowest order approximation compares favorably with our cluster expansion results where $v_{\text {Hahn }}(\tau) \ll 1$. We can be slightly more sophisticated with this small $\tau$ approximation by applying it within the context of the cluster expansion; noting that $v_{\mathcal{C}}^{\prime}(\tau)=O\left(\tau^{4}\right)$, Eq. (11) implies that $v_{\text {Hahn }}(\tau)=$ $\exp \left[-O\left(\tau^{4}\right)\right]$. As discussed in previous work, ${ }^{14}$ convergence for large systems (i.e., a large bath) is attained in this way by embedding the $\tau$ expansion, which is not necessarily convergent for large systems, within the context of the cluster expansion.

In the lowest order $\tau$ approximation discussed above, the GaAs:P result becomes

$$
\ln \left[v_{\mathrm{Hahn}}(\tau)\right] \approx-\left(\frac{\tau}{260 \mu \mathrm{S}}\right)^{4} .
$$

The exact (convergent) results plotted in Fig. 1 do not visibly differ from Eq. (12); therefore this time expansion approximation is valid in the region in which the cluster expansion converges. By applying this lowest order time expansion approximation, as well as the lowest order in the cluster expansion itself (involving only pair contributions), the $\mathrm{Si}: \mathrm{P}$ result becomes

$$
\ln \left[v_{\mathrm{Hahn}}(\tau)\right] \approx-f^{2}\left(\frac{\tau}{1.05 \mathrm{~ms}}\right)^{4} .
$$

The $f^{2}$ dependence simply arises from the fact that, in this approximation, all contributions are from pairs of nuclei. Dotted lines in Figs. 2 and 3 show the lowest order approximation of Eq. (13) for the Hahn echo (as well as the lowest order approximation for the CPMG pulse sequence which will be discussed in the next section). The exact (convergent) results exhibit a slight disagreement with the lowest order approximation as the cluster expansion nears the point of its divergence.

In previous studies of electron spin dephasing ${ }^{14,15}$ we noted good convergence of the cluster expansion for the full decay of the echoes as a result of a particularly applicable intrabath perturbation that treats the coupling between members of the bath as a perturbation in the Hamiltonian. In that situation, the hyperfine coupling between the electron and the nuclei was much stronger than the dipolar coupling among nuclei. In the case of nuclear spin dephasing, however, the coupling to our central nucleus is no stronger than the coupling between members of the bath. In fact, the effective smallness of $\tau$ (compared to the inverse energies of the problem) is apparently the only reason for any cluster expansion convergence in the current study. As a consequence, the cluster expansion convergence is only attainable for short times (small values of $\tau$ ) as indicated in Figs. 1, 3. However, we do obtain convergent and accurate results of the initial quantum memory loss in the duration of high fidelity qubit memory retention which is of greatest significance in the context of quantum computing.

\section{MULTIPLE PULSE REFOCUSING}

It is well known in the NMR community that multiple pulse sequences may be used to prolong qubit coherence for a much greater time than the simple Hahn echo sequence. The simplest of these multiple pulse sequences is known as the Carr-Purcell-Meiboom-Gill $(\mathrm{CPMG})^{16}$ scheme in which the Hahn echo sequence is simply repeated: $(\tau \rightarrow \pi \rightarrow \tau)^{n}$. In our previous work $^{15}$ on electron spin coherence in semiconductors, independently confirmed in Ref. 17, we reported that loworder symmetry-related cancellations occur when an even 
number of CPMG pulses are applied; this leads to significantly longer coherence times of even-pulse CPMG echoes compared with the Hahn echo. Also, increasing the number of these pulses will increase the overall coherence time with the assumption that the applied pulses are ideal. Consistent with these previous findings, we find that donor nucleus coherence is significantly enhanced by applying a two-pulse CPMG relative to the single pulse Hahn refocusing, and further enhanced, in overall coherence time, by applying a four-pulse CPMG sequence. We now consider the application of multiple pulse echo sequences in prolonging nuclear spin quantum memory in semiconductor quantum computer architectures.

If we apply $2 \nu$ CPMG pulses, $(\tau \rightarrow \pi \rightarrow \tau)^{2 \nu}$, the total echo time for this is $t=4 \nu \tau$, and the exact echo envelope may be expressed [analogous to Eq. (5)] as $\underline{15}$

$$
v_{\mathrm{CPMG}}(\nu, \tau)=\left\langle\left[U_{\mathrm{CPMG}}^{-}(\nu, \tau)\right]^{\dagger} U_{\mathrm{CPMG}}^{+}(\nu, \tau)\right\rangle,
$$

where $U_{\mathrm{CPMG}}^{ \pm}(\nu, \tau)$ are nuclear evolution operators in the context of a CPMG echo sequence with an initially up $(+)$ or down $(-)$ electron spin such that

$$
U_{\mathrm{CPMG}}^{ \pm}(\nu, \tau)=\left[U_{0}^{ \pm}(\tau) U_{0}^{\mp}(2 \tau) U_{0}^{ \pm}(\tau)\right]^{\nu}
$$

referring to the definition of $U_{0}^{ \pm}$given in Eq. (7). The angle brackets, $\langle\ldots\rangle$, of Eq. (14) represent a quantum mechanical average over initial bath states that we take to be in a uniform distribution representing a completely disordered bath as we did in Sec. II.

The cluster expansion is performed as described in the previous section using Eqs. (9) and (11) with the only exception that $v_{\mathcal{C}}(\tau)$ should now represent the solution, while considering only the nuclei in cluster $\mathcal{C}$, to Eq. (14). We have performed these cluster expansion calculations to successively approximate Eq. (14) for the GaAs:P and Si:P systems. We again plot "memory loss" versus total echo time $(4 \nu \tau)$ where memory loss is defined as $1-v_{\mathrm{CPMG}}(\tau)$. Results for 2 and 4 pulses $(\nu=1$ and 2 , respectively) in the GaAs:P system are shown in Fig. 1 where they are compared with Hahn echo results. Similarly, results for the natural and isotopically purified Si:P systems are, respectively, shown in Figs. 2 and 3 where they are also compared with corresponding Hahn echo results. A spin coherence enhancement when applying two pulses versus one pulse (Hahn echo) is apparent in the plots for each of these systems.

As shown in Ref. 15, if we apply a lowest order time expansion and an intrabath perturbation (with coupling between nuclei as a perturbation in the Hamiltonian) approximation in the cluster contribution terms that go into the cluster expansion calculation, we find that $v_{\mathrm{CPMG}}(\tau) \sim \exp \left[-O\left(\nu^{2} \tau^{6}\right)\right]=\exp \left[-O\left(\nu^{-4} t^{6}\right)\right]$. In this lowest order approximation, the general GaAs:P result

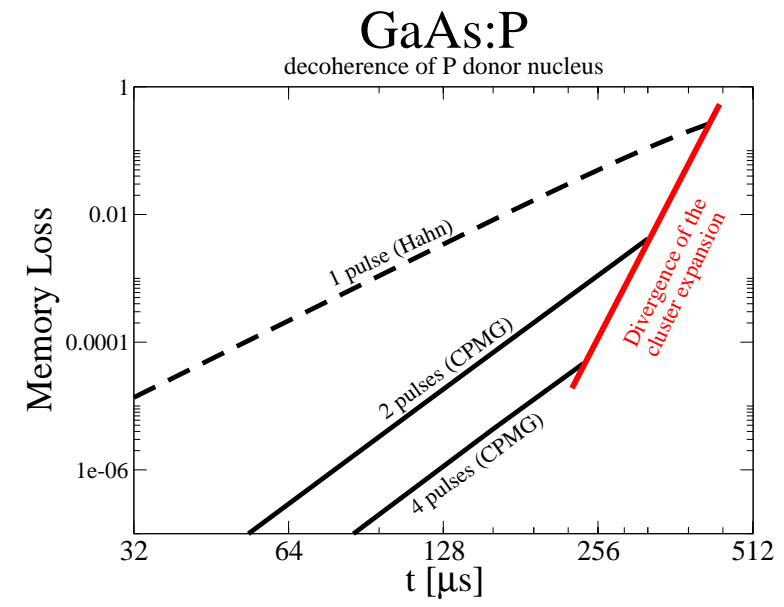

FIG. 1: Numerical results of nuclear spin quantum memory loss for a ${ }^{31} \mathrm{P}$ donor nucleus that replaces an As atom in bulk GaAs. We define memory loss as one minus the normalized echo and plot this in a log-log scale as a function of the total echo time. The dashed line gives the Hahn echo results and the solid lines give CPMG echo results for two and four pulses. At some point for each type of echo sequence, the cluster expansion fails to converge.

becomes

$$
\begin{aligned}
\ln \left[v_{\mathrm{CPMG}}(\tau)\right] & \approx-\nu^{2}\left(\frac{\tau}{195 \mu \mathrm{s}}\right)^{6} \\
& =-\nu^{-4}\left(\frac{t}{780 \mu \mathrm{s}}\right)^{6}
\end{aligned}
$$

as compared with $\ln \left[v_{\text {Hahn }}(\tau)\right] \approx-(t / 520 \mu \mathrm{s})^{4}$ [Eq. (12) rewritten in terms of $t]$. The exact (convergent) results plotted in Fig. 11 do not visibly differ from Eq. (16); therefore this approximation is valid in the region in which the cluster expansion converges. This short time approximation equation may serve as a useful educated guess (estimate) at times beyond cluster expansion convergence although the decay is expected to eventually exhibit exponential, Markovian behavior. If we do extrapolate Eq. (16) and define $T_{2}$ as the time in which the extrapolated echo reaches $1 / e$, then we have $T_{2}=\nu^{0.67} \times 780 \mu \mathrm{s}$ for even CPMG echoes. This gives a factor of 6.5 increase of nuclear spin coherence times relative to the electron spin quantum dot coherence times reported in Ref. 15.

In the range of cluster convergence, where we have confidence in the accuracy of our results for the model that we have used, we observe high fidelity memory retention with a low loss of $10^{-6}$ up to $80-120 \mu$ s for two or four-pulse CPMG sequences. Increasing the number of CPMG pulses can improve fidelity versus time theoretically; however, in practice one will be limited by the finite time required to perform each $\pi$-pulse and by the accumulation of errors resulting from multiple pulses. Because our analysis assumes that each pulse is ideal, which is unlikely in practice, these theoretical results for $T_{2}$ should thus be regarded as an upper bound on the 


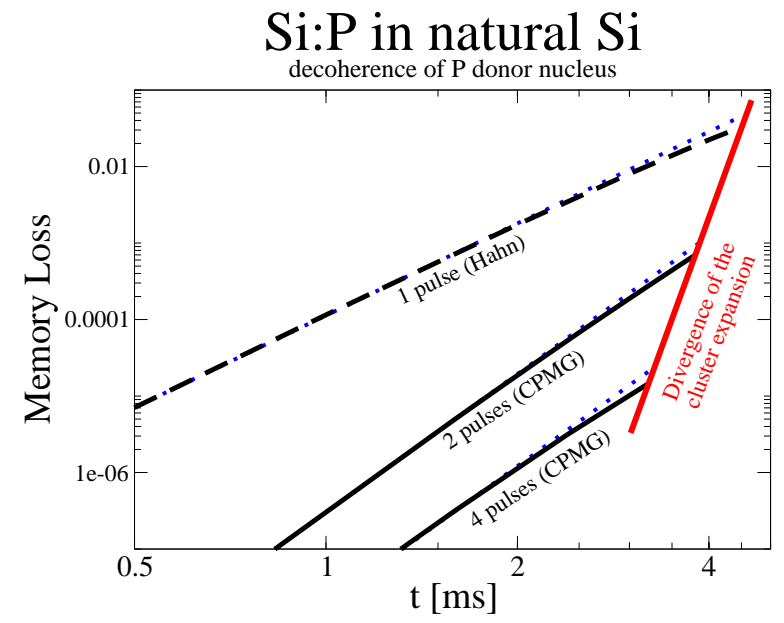

FIG. 2: Numerical results of nuclear spin quantum memory loss for a ${ }^{31} \mathrm{P}$ donor nucleus in bulk $\mathrm{Si}$. We define memory loss as one minus the normalized echo and plot this in a log-log scale as a function of the total echo time. The dashed line gives the Hahn echo results and the solid lines give CPMG echo results for two and four pulses. Dotted lines give corresponding results, for comparison, obtained from the lowest order expansions provided by Eqs. (13) and (17). At some point for each type of echo sequence, the cluster expansion fails to converge.

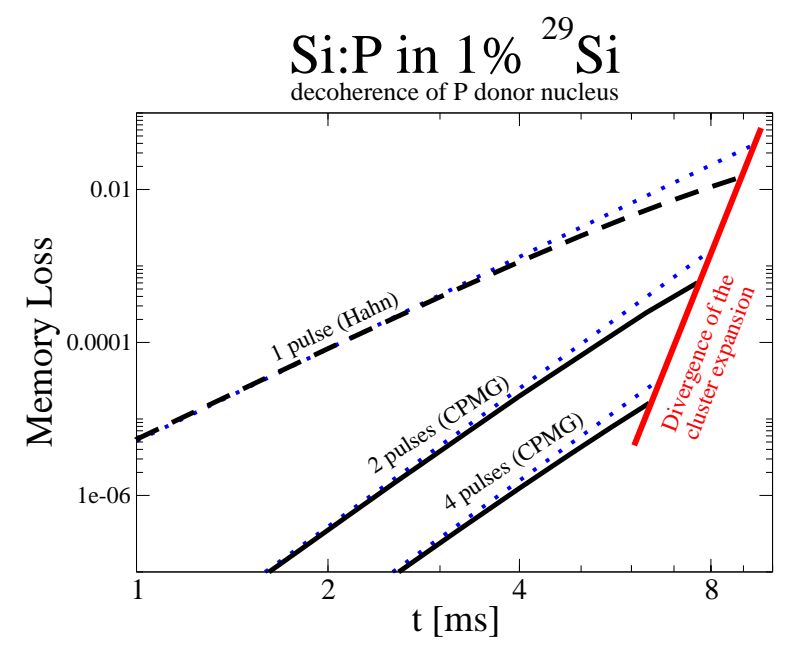

FIG. 3: Equivalent to Fig. 2 except that results are shown for Si purified to $1 \%{ }^{29} \mathrm{Si}$. Lowest order results given by Eqs. (13) and (17) are shown by the dotted lines. Isotopic purification enhances coherence as predicted in these equations.

nuclear memory time.

Applying the lowest order approximation to the Si:P system, we similarly obtain

$$
\begin{aligned}
\ln \left[v_{\mathrm{CPMG}}(\tau)\right] \approx & -\nu^{2} f^{2}\left[\left(\frac{\tau}{1.35 \mathrm{~ms}}\right)^{6}+f\left(\frac{\tau}{0.70 \mathrm{~ms}}\right)^{6}\right] \\
= & -\nu^{-4} f^{2}\left(\frac{t}{5.4 \mathrm{~ms}}\right)^{6} \\
& -\nu^{-4} f^{3}\left(\frac{t}{2.8 \mathrm{~ms}}\right)^{6} .
\end{aligned}
$$

as compared with $\ln \left[v_{\text {Hahn }}(\tau)\right] \approx-f^{2}(t / 2.1 \mathrm{~ms})^{4}$ [Eq. (13) rewritten in terms of $t$ ]. These lowest order results are shown as dotted lines in Figs. 2 and 3 . The exact (convergent) results initially match this lowest order approximation, but show a slight disagreement as the cluster expansion nears the point of its divergence.

As with Eqs. (12) and (16), the above equations may serve as a useful educated guess (estimate) at times beyond cluster expansion convergence. Initially, at least, Figs. 2 and 3 show that Eqs. (13) and (17) provide conservative estimates. If we do extrapolate these equations and define $T_{2}$ as the time in which the extrapolated echo reaches $1 / e$, then we have, for natural $\mathrm{Si}$, $T_{2}=\nu^{0.67} \times 12 \mathrm{~ms}$ for even CPMG echoes. For a small number of pulses, $\nu \sim 1$, this gives about a factor of 5 increase of nuclear spin coherence times relative to the electron spin quantum dot coherence times reported in Ref. 15; this comparison factor increases as we increase the number of pulses because electron spin decay time 15 scales with a smaller power of $\nu\left(\nu^{0.53}\right)$. In the range of cluster convergence, where we have confidence in the accuracy of our results for the model that we have used, we observe high fidelity memory retention with a low loss of $10^{-6}$ up to $1-2 \mathrm{~ms}$ for two or four-pulse CPMG sequences in natural $\mathrm{Si}$ and nearly up to $4 \mathrm{~ms}$ for $1 \%$ purified Si.

By implementing CPMG pulse sequences with just a few even number of pulses, high fidelity (with loss below $10^{-6}$ ) qubit retention times are theoretically observed on the order of $100 \mu \mathrm{s}$ for GaAs systems and on the order of milliseconds for Si:P systems. We emphasize that although we are unable to achieve convergence beyond the initial decay (for reasons discussed at the end of Sec. II) which affects the accuracy of our extrapolated estimate for $T_{2}$, itself, we accurately estimate the initial-time coherent memory loss (i.e., the loss of the first $10^{-4}-10^{-6}$ fraction of coherence) which is the most important ingredient for quantum computation considerations.

\section{INTRINSIC SPIN RELAXATION}

In addition to storing quantum information on individual nuclear spins, there is also a proposa ${ }^{15}$ to store quantum information in coherent states of nuclear spin ensembles. The proposal suggests transferring the spin state of a quantum dot electron onto coherent spin states of the nuclei within the dot via resonance. Before the information is transferred, the nuclei must be polarized or 
cooled into a so-called dark state that will only interact with electrons of one type of polarization and not the other; this can be accomplished by injecting polarized electrons into the dot.18 Then through the hyperfine interactions between the electron and the nuclei, one may induce Rabi oscillations via resonant tuning of the external magnetic field that will pass the quantum information back and forth between the electron spin and coherent states of the nuclear ensemble. In this way, one can transfer the quantum information from the electron spin to the nuclear spins. The electron can then be moved off of the dot, and the nuclear ensemble will store the quantum state for milliseconds 19 until it is retrieved back onto an electron spin state by the same process using Rabi oscillations.

The coherent state of the nuclear spin ensemble in such a proposal can be destroyed by nuclear spin dynamics induced, for example, by dipolar interactions between nuclear spins. If an electron is kept on the dot during the storage period, differences of hyperfine energies will suppress dipolar interactions that lead to decoherence; however, these differences of hyperfine energies in themselves result in an even more rapid loss of coherence due to developing phase differences of the nuclear spins 19 Thus it is best to move the electron off of the dot; then the quantum memory time for this proposal will be determined solely by interactions (e.g., dipolar) among the nuclei. Assuming that the quantum state is imprinted on a nuclear spin ensemble that is fully polarized initially, the stored nuclear state will be a superpositon of the fully polarized state and each possible state with only one spin oppositely polarized; then the coherent state is lost, in particular, as the oppositely polarized nucleus, of the various superposition states, is transported off of the dot by dipolar flip-flop processes. This situation is analyzed for specific quantum dot geometries in Ref. 19.

We will study the problem of the decoherence of such a nuclear spin ensemble state in a more general and simple way that provides a lower bound limit of coherence time (in the ideal case). Instead of considering a specific dot geometry and studying decoherence as a process in which spins are transported off of the dot, we simply consider the spin relaxation of a single nuclear spin in a bath of fully polarized spins. This provides a lower bound, or pessimistic estimate, of the coherence time of an ensemble nuclear spin state such as the previous proposal and gives a general consideration for similar proposals that may arise. This simple analysis also gives a nice theoretical counterpart to spectral diffusion which is not possible in this scenario; in the limit of a strong applied magnetic field flip-flop processes cannot occur among spins of the polarized bath.

With this motivation, we study a well-defined problem to compute the lifetime of quantum information stored in a central "intrinsic" (as opposed to a donor) nuclear spin, surrounded by a bath of fully polarized nuclei of the same type, and only considering nuclear spin degrees of freedom in the limit of a strong applied magnetic field.
The precise scenario being considered here in the context of nuclear spin quantum memory lifetime is the following: The central spin in a polarized nuclear spin bath has its spin opposite to all the other spins in the bath initially and we are interested in finding out how this local spin excitation moves (i.e., "diffuses," although the process is technically not a diffusive process) away from the central spin due to dipolar coupling with surrounding nuclei. The central spin serves as the quantum memory which "decays" in time as the state evolves into superpositions of excitation states involving spins in the bath. The effective Hamiltonian for the dipolar interaction, under the influence of a strong magnetic field which effectively conserves polarization via energy conservation, is given by

$$
\mathcal{H}=\sum_{n \neq m} b_{n m}\left(I_{n+} I_{m-}-2 I_{n z} I_{m z}\right)
$$

where the $I_{n}$ operators operate on the nuclear spin at site $n$ in the basis in which $z$ is in the direction of the applied magnetic field. The dipolar coupling is given by

$$
b_{n m}=-\frac{1}{4} \gamma_{I}^{2} \hbar^{2} \frac{1-3 \cos ^{2} \theta_{n m}}{R_{n m}^{3}},
$$

where $\theta_{n m}$ is the angle formed between the applied magnetic field and the bond vector linking the two spins. In a uniform applied magnetic field, which we assume, the total Zeeman energy will be constant when polarization is conserved. Therefore the magnetic field does not affect the dynamics beyond determining the preferred $z$ direction and justifying the polarization conservation approximation of Eq. (18).

In our initial state, we will assume a fully polarized nuclear spin bath. We denote the fully polarized state, including the polarization of the central spin, as $|\uparrow N\rangle$. We denote the state with the spin of nucleus $n$ lowered once (via the lowering spin operator) from its fully polarized state, but the remaining nuclei polarized, as $|n\rangle=I_{n-}\left|\uparrow^{N}\right\rangle$. We can store a qubit in the superposition of states $\left|\uparrow^{N}\right\rangle$ and $|0\rangle$. The state $\left|\uparrow^{N}\right\rangle$ is an eigenstate of Eq. (18) and is therefore stable. The $|0\rangle$ state, however, can decay as a result of a flip-flop with some neighboring nucleus; in this section, we will determine the lifetime of the $|0\rangle$ state for two different types of materials, GaAs and $\mathrm{Si}$, and characterize the decay curve. We can solve this problem exactly by using a Hilbert space composed of the $|n\rangle$ states as a basis (since our Hamiltonian does not include any interactions that can take us out of this state space). Conveniently, this state space grows linearly with the number of nuclei (rather than the exponential growth of the Hilbert space that occurs in general) which allows this problem to be solved exactly even when there are many neighbors that influence the decay.

We wish to directly compute the probability that the system remains in state $|0\rangle$ after allowing the system to freely evolve for a time $t$ :

$$
P_{0}(t)=\|\langle 0|\exp (-i \mathcal{H} t)| 0\rangle\|^{2} .
$$


To solve this problem directly, we first compute the matrix elements of $\mathcal{H}$ in the $|n\rangle$ basis and then we can exponentiate this matrix via direct diagonalization. The off-diagonal elements of $\mathcal{H}$ are

$$
\langle n|\mathcal{H}| m\rangle=2 I b_{n m}, \forall n \neq m
$$

where $I$ is the nuclear spin magnitude, assumed to be the same for all involved nuclei. To obtain this we have used

$$
I_{ \pm}|I, m\rangle=\sqrt{I(I+1)-m(m \pm 1)}|I, m \pm 1\rangle,
$$

where only $I_{+}|I, I-1\rangle$ and $I_{-}|I, I\rangle$ are relevant. We also have

$$
\begin{aligned}
\langle n|\mathcal{H}| n\rangle & =-2 \sum_{k \neq m} b_{k m} I\left(I-\delta_{n k}-\delta_{n m}\right) \\
& =-2 \sum_{k \neq m} b_{k m} I^{2}+4 \sum_{m \neq n} b_{n m} I .
\end{aligned}
$$

The first term in Eq. (24) is a constant contribution to the diagonal and therefore a constant, commuting contribution to the Hamiltonian that will not affect probabilities. We can therefore use an effective Hamiltonian, $\mathcal{H}^{\prime}$, in which

$$
\begin{aligned}
\left\langle n\left|\mathcal{H}^{\prime}\right| m\right\rangle & =2 I b_{n m}, \forall n \neq m, \\
\left\langle n\left|\mathcal{H}^{\prime}\right| n\right\rangle & =4 I \sum_{n \neq m} b_{n m} .
\end{aligned}
$$

We may now solve the problem by successively including increasingly distant neighbors of the central spin into the simulated system until convergence is achieved. In a Bravais lattice with only one type of nucleus, $\left\langle n\left|\mathcal{H}^{\prime}\right| n\right\rangle$ [Eq. (26)] is, by symmetry, a constant for all $n$ and is therefore irrelevant (another constant energy in the system that plays no role in the dynamics). In lattices with mixed nuclei, this may not be completely irrelevant; however, we have performed calculations on real lattice systems with and without this part of the Hamiltonian finding that it has little effect on the results except that it artificially slows down the rate of convergence as we include increasingly distant neighbors (because it does lead to a significant finite size effect).

Calculated results are shown for pure ${ }^{29} \mathrm{Si}$ and natural $\mathrm{Si}$ in Figs. 4 and 5 , respectively. The ${ }^{29} \mathrm{Si}$ has a spin of $1 / 2$ and $\gamma_{I}=5.31 \times 10^{3}(\mathrm{~s} \mathrm{G})^{-1}$. The other stable isotopes of $\mathrm{Si},{ }^{28} \mathrm{Si}$ and ${ }^{30} \mathrm{Si}$, have no net nuclear spin and therefore do not contribute to the decay. Si has a diamond lattice structure with a lattice constant of $5.43 \AA$. In natural $\mathrm{Si}$, there is a $4.67 \%$ abundance of ${ }^{29} \mathrm{Si}$ and the random placement of these nuclei leads to uncertainty in the results; the standard deviation is thus shown by the error bars in Fig. 5.

Calculated results are shown for GaAs in Fig. 6. There are three distinct problems with the central spin being ${ }^{75} \mathrm{As},{ }^{69} \mathrm{Ga}$, or ${ }^{71} \mathrm{Ga}$ which have respective gyromagnetic ratios of $\gamma_{I}=4.58,6.42$, and $8.16 \times 10^{3}(\mathrm{~s} \mathrm{G})^{-1}$ and all have spins of $3 / 2$. The GaAs is in a zinc-blende lattice structure with a lattice constant of $5.65 \AA$. The

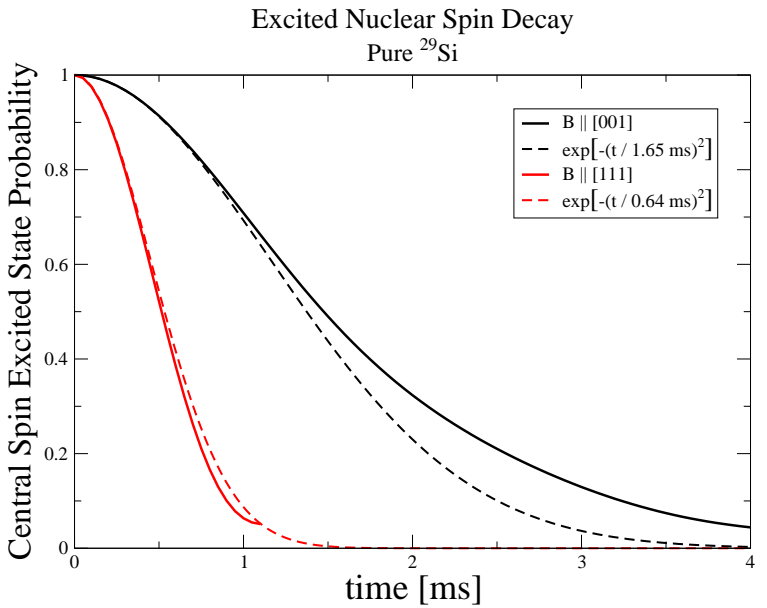

FIG. 4: Relaxation of a central ${ }^{29} \mathrm{Si}$ nuclear spin in a bath of polarized isotopically pure ${ }^{29} \mathrm{Si}$ nuclei in silicon's diamond lattice structure in the limit of a strong applied magnetic field along two directions with extremal results: $B$ along [001] (or, equivalently, along any of the conventional axes, $x, y$, or $z$ ) and $B$ along [111]. The dashed curves give the corresponding approximate solutions from Eq. (31).

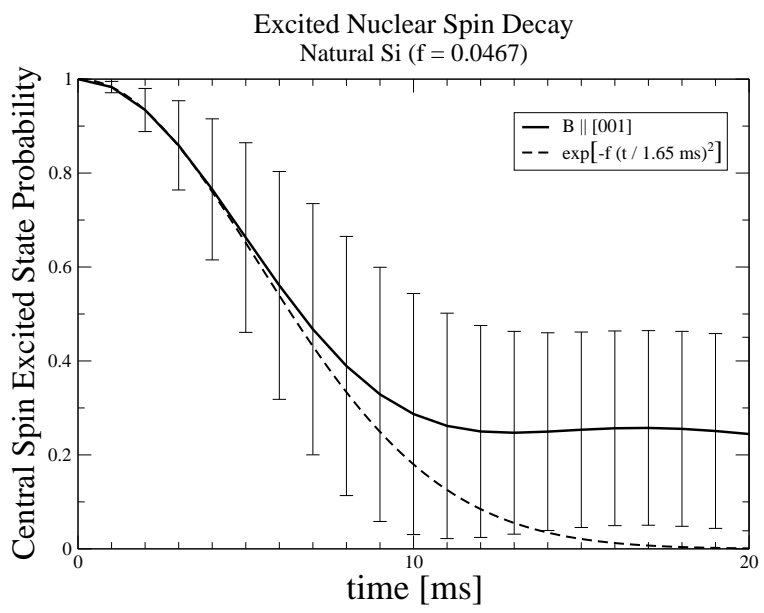

FIG. 5: Relaxation of a central ${ }^{29} \mathrm{Si}$ nuclear spin in a bath of polarized natural Si nuclei in silicon's diamond lattice structure in the limit of a strong applied magnetic field along one of the conventional axes (e.g., [100]). Error bars indicate the standard deviation as a result of random isotopic configurations $\left(4.67 \%{ }^{29} \mathrm{Si}\right.$ and the remaining ${ }^{28} \mathrm{Si}$ and ${ }^{30} \mathrm{Si}$ is spinless giving no contribution to the relaxation). The dashed curve gives the corresponding approximate solution from Eq. (31).

${ }^{75} \mathrm{As}$ occupies one fcc lattice while the ${ }^{69} \mathrm{Ga}$ and ${ }^{71} \mathrm{Ga}$ are randomly distributed in $60.4 \%$ and $39.6 \%$ respective concentrations on the other fcc lattice. The uncertainty in the random isotopic configuration of the Ga sublattice is the cause for standard deviations shown by the error bars in Fig. 6.

To approximate the short time behavior, let us first expand to the lowest order in powers of $t$. First note that $\left\langle 0\left|H^{\prime}\right| 0\right\rangle=0$ so there is not a first power of $t$ in 


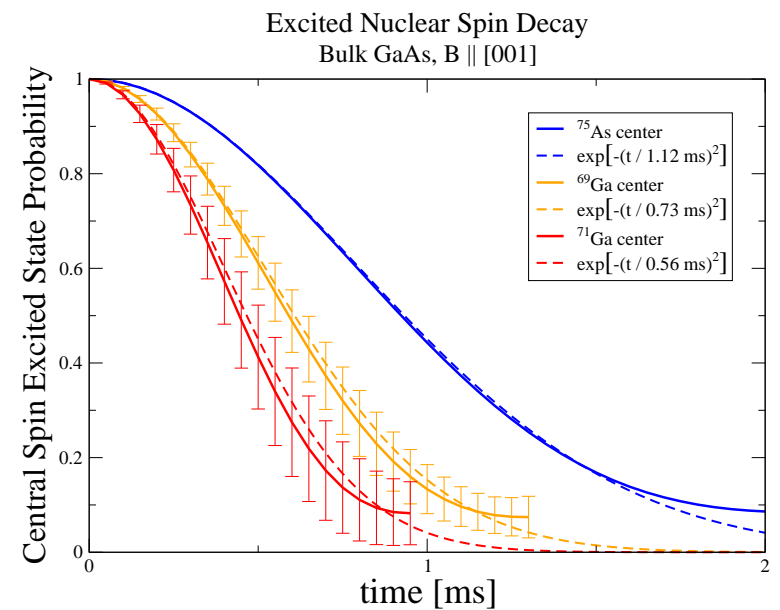

FIG. 6: Relaxation of a central ${ }^{75} \mathrm{As},{ }^{69} \mathrm{Ga}$, or ${ }^{71} \mathrm{Ga}$ nuclear spin in a bath of polarized GaAs nuclei in GaAs's zinc-blende lattice structure in the limit of a strong applied magnetic field along one of the conventional axes (e.g., [100]). In this limit, the central spin only interacts with like nuclei. The ${ }^{75}$ As are arranged with certainty on one of the fcc lattices, while the ${ }^{69} \mathrm{Ga}$ and ${ }^{71} \mathrm{Ga}$ randomly occupy $60.4 \%$ and $39.6 \%$ of the other fcc lattice, respectively. Error bars for the two Ga results indicate the standard deviation as a result of random isotopic configurations. The dashed curves give the corresponding approximate solutions from Eq. (31).

the expansion. In order to obtain the next order (first nontrivial order), we have

$$
\begin{aligned}
\left\langle 0\left|\left(\mathcal{H}^{\prime}\right)^{2}\right| 0\right\rangle & =\sum_{n}\left\langle 0\left|\mathcal{H}^{\prime}\right| n\right\rangle\left\langle n\left|\mathcal{H}^{\prime}\right| 0\right\rangle \\
& =4 I^{2} \sum_{n \neq 0} b_{n 0}^{2},
\end{aligned}
$$

so then

$$
\langle 0|\exp (-i \mathcal{H} t)| 0\rangle \approx 1-2 I^{2} \sum_{n \neq 0} b_{n 0}^{2} t^{2} .
$$

Squaring this and keeping only terms up to $t^{2}$, we finally obtain

$$
P_{0} \approx 1-(2 I)^{2} \sum_{n \neq 0} b_{n 0}^{2} t^{2}
$$

To better match the shape of the decay curves, we use an exponential in time that is consistent with the above approximation:

$$
P_{0} \approx \exp \left(-f(2 I)^{2} \sum_{n \neq 0} b_{n 0}^{2} t^{2}\right) .
$$

In order to effectively average over isotopic configurations, we have now explicitly inserted the dependence on the fraction, $f$, of nuclei in the lattice (or sublattice) that is of the same type as the central spin. This equation is indeed a good approximation to the initial decay and is exhibited by the dashed curves in Figs. 4, 5, and 6, A good characterization of the lifetime is the time in which this approximation reaches $1 / e$ which is given by

$$
t_{0}=1 / \sqrt{f(2 I)^{2} \sum_{n \neq 0} b_{n 0}^{2}} .
$$

This also indicates how the lifetime scales with isotopic purification (e.g., in $\mathrm{Si}$ ). Using this $t_{0}$ to define the lifetime, natural $\mathrm{Si}$ has a lifetime of $1.7 \mathrm{~ms}$, and for GaAs, ${ }^{75} \mathrm{As},{ }^{69} \mathrm{Ga}$, and ${ }^{71} \mathrm{Ga}$ have lifetimes of $1.1,0.7$, and $0.6 \mathrm{~ms}$, respectively. Note that while there is a greater abundance of ${ }^{75}$ As than the other nuclear types in GaAs, it has a longer lifetime due to its relatively small gyromagnetic ratio.

\section{CONCLUSION}

We have analyzed in this work two complementary theoretical issues regarding the prospect for using nuclear spins in semiconductor materials, specifically GaAs and $\mathrm{Si}$ (with and without $\mathrm{P}$ doping), as long-lived quantum memory. We find in general that solid state nuclear quantum memory time could be one to two orders of magnitude longer than the corresponding electron spin coherence times. Thus using nuclear spins as quantum memory in semiconductor nanostructures may be useful in the context of solid state quantum information processing architectures.

We address two complementary situations of solid state nuclear spin coherence, both taken in the limit of a strong applied magnetic field: (1) quantum memory stored in a donor nuclear spin in a surrounding unpolarized nuclear spin bath where the main memory loss (i.e., decoherence) occurs through the spectral diffusion mechanism associated with the non-Markovian temporally fluctuating random magnetic field created by the dipolar flip-flops in the nuclear spin bath (Secs. II and III); and (2) quantum memory stored in an intrinsic spin surrounded by a spinpolarized nuclear bath where the spin relaxes through direct flip-flop interactions with neighboring nuclei of the same type (Sec. IV). These are mutually exclusive situations; spectral diffusion disregards spin relaxation (justified in a strong magnetic field), and a polarized bath cannot induce spectral diffusion.

In case (1), we note that our $T_{2}$ dephasing times define characteristic time scales for the initial part of the decay that is governed by non-Markovian dynamics in the bath. Our $T_{2}$ values of $520 \mu \mathrm{s}$ for $\mathrm{P}$ in GaAs and $9.7 \mathrm{~ms}$ for $\mathrm{P}$ in natural $\mathrm{Si}$ are consistent with the long-time exponential decay behavior studied in NMR,,$\underline{12}$ but it is important to note that we expect a different short time echo decay behavior of the form $\exp \left[-\left(t / T_{2}\right)^{4}\right]=\exp \left[-\left(2 \tau / T_{2}\right)^{4}\right]$ for the Hahn sequence. This has consequences for the nature of high-fidelity quantum memory retention that is most important for quantum computing considerations. 
Longer memory times may be achieved in case (1) by using composite CPMG pulse sequences; with an even number of $2 \nu$ pulses and total sequence time $t=4 \nu \tau$, the echo decay behavior is of the form $\exp \left[-\nu^{2}\left(\tau / \tau_{0}\right)^{6}\right]=$ $\exp \left[-\nu^{-4}\left(t / t_{0}\right)^{6}\right]=\exp \left[-t^{2} \tau^{4} /\left(16 \tau_{0}^{6}\right)\right]$. The latter expression is relevant to a train of pulses with fixed $\tau$; because we consider the non-Markovian, nonexponential regime of initial decay, there is no single $T_{2}$ for a train of pulses that is independent of $\tau$. Our figures show considerable coherence enhancement with just two and four applied pulses. Further enhancement can be achieved by applying more pulses, but this has the cost of requiring more frequent pulses that must be applied precisely, which has physical limitations. Isotopic purification can further extend coherence in Si by eliminating ${ }^{29} \mathrm{Si}$ nuclei $\left({ }^{28} \mathrm{Si}\right.$ and ${ }^{30} \mathrm{Si}$ nuclei have no free spin moments); with $f$ as the fraction of ${ }^{29} \mathrm{Si}$ in the bath, we find that $T_{2} \propto 1 / \sqrt{f}$ (or $T_{2} \propto f^{-1 / 3}$ when pair contributions dominate the decay of the CPMG sequence). In principle, it is possible to extend quantum memory time in Si indefinitely by eliminating all ${ }^{29} \mathrm{Si}$, but this is not possible in GaAs where all isotopes have nonzero spin.

When quantum memory is stored on a nucleus that is identical to the nuclei in the surrounding bath (rather than a donor), relaxation is not suppressed by applying a strong magnetic field and spectral diffusion is somewhat ambiguous because the concept of the central spin is ill- defined. In case (2), we have chosen to study relaxation of an intrinsic nucleus in a bath of polarized nuclear spins in which spectral diffusion is suppressed. We find that this relaxation decay takes the form of $\exp \left[-\left(t / T_{1}\right)^{2}\right]$; we find relaxation times in GaAs of $T_{1}=0.6,0.7$, and $1.1 \mathrm{~ms}$ for a central spin of ${ }^{71} \mathrm{Ga},{ }^{69} \mathrm{Ga}$, or ${ }^{75} \mathrm{As}$, respectively, and relaxation times in $\mathrm{Si}$ of $T_{1}=1.7 \mathrm{~ms} / \sqrt{f}$ with $f$ as the fraction of ${ }^{29} \mathrm{Si}$ ( $T_{1} \sim 8 \mathrm{~ms}$ for natural $\mathrm{Si}$ in particular). These results are relevant, for example, to the proposal ${ }^{\underline{5}}$ of quantum memory storage in nuclear spin ensembles within quantum dots. We do not consider loss of memory due to moving an electron on and off the quantum dot. We also do not treat the precise dot-dependent decoherence in such ensembles that results from spins "leaking" off the quantum dot; such a consideration is made in Ref. 19. The virtue of our analysis is in its simplicity and generality. By considering the rate at which a spin moves away from a central nucleus, we provide a lower bound for the coherence times in any such nuclear spin memory proposal in the ideal case where dipolar interactions among the nuclei dominate decoherence and the bath is polarized (otherwise, spectral diffusion also plays a role). We study this well-defined problem using exact quantum mechanical calculations (i.e., diagonalizing the Hamiltonian).

This work was supported by ARO-DTO, ARO-LPS, and NSA-LPS.
1 B. E. Kane, Nature (London) 393, 133 (1998); A. J. Skinner, M. E. Davenport, and B. E. Kane, Phys. Rev. Lett. 90, 087901 (2003).

2 T. D. Ladd, D. Maryenko, Y. Yamamoto, E. Abe, and K. M. Itoh, Phys. Rev. B 71, 014401 (2005).

3 L. Childress, J. M. Taylor, A. S. Sorensen, and M. D. Lukin, Phys. Rev. Lett. 96, 070504 (2006).

${ }^{4}$ P. van Loock, T. D. Ladd, K. Sanaka, F. Yamaguchi, K. Nemoto, W. J. Munro, and Y. Yamamoto, Phys. Rev. Lett. 96, 240501 (2006).

5 J. M. Taylor, C. M. Marcus, M. D. Lukin, Phys. Rev. Lett. 90, 206803 (2003).

6 A. Abragam, The Principles of Nuclear Magnetism (Oxford University Press, London, 1961); M. Levitt, Spin Dynamics: Basics of Nuclear Magnetic Resonance (Wiley, New York, 2001); R. Freeman, Spin Choreography: Basic Steps in High Resolution NMR (Oxford University Press, New York, 2004).

7 M. A. Nielsen and I.L. Chuang, Quantum Computation and Quantum Information (Cambridge University Press, Cambridge, UK, 2000).

8 Xuedong Hu and S. Das Sarma, Phys. Status Solidi B 238, 360 (2003); S. Das Sarma et al., Solid State Commun. 133, 737 (2005).

9 H. Y. Carr and E. M. Purcell, Phys. Rev. 94, 630 (1954); S. Meiboom and D. Gill, Rev. Sci. Instrum. 29, 6881 (1958).

10 J. S. Waugh, L. M. Huber, and U. Haeberlen, Phys. Rev. Lett. 20, 180 (1968).

11 J. Preskill, Proc. R. Soc. London, Ser. A 454, 385 (1998); P. Aliferis, D. Gottesman, and J. Preskill, Quantum Inf. and Comput. 6, 97 (2006); K. M. Svore, D. P. DiVincenzo, and B. M. Terhal, arXiv:quant-ph/0604090, T. Szkopek et al., IEEE Trans. Nanotechnol. 5, 42 (2006); D. Gottesman, arXiv:quant-ph/0701112.

12 D. Paget et al., Phys. Rev. B 15, 5780 (1977); R. K. Hester et al., ibid. 10, 4262 (1974); G.P. Carver, D.F. Holcomb, J.A. Kaeck, ibid. 3, 4285 (1971); R. K. Sundfors, Phys. Rev. 185, 458 (1969); R. K. Sundfors and D. F. Holcomb, Phys. Rev. 136, A810 (1964).

13 A. E. Dementyev, D. Li, K. MacLean, and S. E. Barrett, Phys. Rev. B 68, 153302 (2003); S. Watanabe and S. Sasaki, Jpn. J. Appl. Phys., Part 2 42, L1350 (2003).

14 W. M. Witzel, R. de Sousa, and S. Das Sarma, Phys. Rev. B 72, 161306(R) (2005); W. M. Witzel and S. Das Sarma, ibid. 74, 035322 (2006).

15 W. M. Witzel and S. Das Sarma, Phys. Rev. Lett. 98, 077601 (2007).

16 The Carr Purcell pulse sequence is more simple than CPMG but they only differ in the initialization pulse that is irrelevant for our purpose of storing any initial state. The CPMG sequence was designed to provide better protection against pulse errors on even echoes. Since we treat only even multiple-pulse echoes, having improved coherence over odd echoes in the presence of the nuclear bath, we use CPMG to denote the sequence.

17 Wang Yao, Ren-Bao Liu, and L. J. Sham, Phys. Rev. B 74, 195301 (2006); Phys. Rev. Lett. 98, 077602 (2007); J. M. Taylor et al., arXiv:cond-mat/0407640

18 A. Imamoğlu, E. Knill, L. Tian, and P. Zoller, Phys. Rev. Lett. 91, 017402 (2003). 
19 Changxue Deng and Xuedong Hu, IEEE Trans. Nanotechnol. 4, 35 (2005). 